\title{
Low-frequency noise in electric double layer InGaZnO thin- film transistors gated with sputtered SiO2-based electrolyte
}

DOI:

10.1021/acsaelm.9b00141

\section{Document Version}

Accepted author manuscript

Link to publication record in Manchester Research Explorer

Citation for published version (APA):

Ma, X., Zhang, J., Cai, W., Wilson, J., \& Song, A. (2019). Low-frequency noise in electric double layer InGaZnO thin-film transistors gated with sputtered SiO2-based electrolyte. ACS Applied Electronic Materials, 1, 972. https://doi.org/10.1021/acsaelm.9b00141

\section{Published in:}

ACS Applied Electronic Materials

\section{Citing this paper}

Please note that where the full-text provided on Manchester Research Explorer is the Author Accepted Manuscript or Proof version this may differ from the final Published version. If citing, it is advised that you check and use the publisher's definitive version.

\section{General rights}

Copyright and moral rights for the publications made accessible in the Research Explorer are retained by the authors and/or other copyright owners and it is a condition of accessing publications that users recognise and abide by the legal requirements associated with these rights.

\section{Takedown policy}

If you believe that this document breaches copyright please refer to the University of Manchester's Takedown Procedures [http://man.ac.uk/04Y6Bo] or contact uml.scholarlycommunications@manchester.ac.uk providing relevant details, so we can investigate your claim.

\section{OPEN ACCESS}




\title{
Graphene bridge rectifier based on self-switching diode arrays
}

\author{
Jiawei Zhang ${ }^{1,3}$, Joseph Brownless ${ }^{1,3}$ and Aimin Song ${ }^{1,2}$ \\ ${ }^{1}$ School of Electrical and Electronic Engineering, University of Manchester, Manchester M13 9PL, \\ United Kingdom \\ ${ }^{2}$ State Key Laboratory of Crystal Materials, Centre of Nanoelectronics and School of Microelectronics, \\ Shandong University, Jinan 250100, People's Republic of China \\ ${ }^{3}$ These authors contributed equally to this work. \\ E-mail: A.Song@manchester.ac.uk
}

Received xxxxxx

Accepted for publication $\mathrm{xxxxx}$

Published xxxxxx

\begin{abstract}
Here, we present theory and measurements for a bridge rectifier formed from arrays of graphene self-switching diodes (GSSDs). Despite graphene's lack of a bandgap and high carrier concentration causing a reduced rectification ratio, the extremely high carrier mobility will allow GSSDs to work at frequencies well into the THz region. Compared with a single SSD array, the bridge rectifier structure allows for full wave rectification of an AC signal. Here we derive an equation for the voltage output of a bridge rectifier formed from GSSDs, which predicts a quadratic relationship between output voltage and input current. This relationship is confirmed using AC and DC measurements. The fabricated rectifier is found to have a high room temperature intrinsic responsivity of 4,395 V/W at low frequency and a low noise equivalent power of $5.4 \mathrm{pW} / \mathrm{Hz}^{1 / 2}$.
\end{abstract}

Keywords: Self-switching diode, graphene, terahertz

\section{Introduction}

The self-switching diode (SSD) is a planar, nanoscale, two terminal device which uses electrostatic effects to achieve asymmetric conduction through a narrow channel. This device produces this rectifying I-V output without the use of any doping junctions or barrier structure, instead using effects arising purely from its geometry $[1,2]$. The SSD was first proposed and fabricated by Song et al. in 2003, demonstrating the rectifying ability of this device in an InGaAs/InAlAs material system [1]. Since then, there have been experiments showing their capability to operate at room temperature at high frequencies, up to and including terahertz (THz) [3]. They have been operated as microwave and $\mathrm{THz}$ detectors [4-6], and fabricated in other materials such as $\mathrm{GaN}$ [5], organic polymer [7], and zinc-oxide thin films [8].
SSDs fabricated from graphene (also known as graphene SSDs, GSSDs) have also been demonstrated [6,9,10], modelled [11], and simulated [12,13]. Due to the capability of SSDs constructed from InGaAs/InAlAs and other materials to operate at $\mathrm{THz}$ frequencies [4], and the greater carrier mobility in graphene, it is expected that GSSDs will also be capable of operating at $\mathrm{THz}$ frequencies. An alternative design of geometric diode has also been demonstrated in graphene by Moddel et al. [14]. While the GSSD operates using only electrostatic effects arising from geometry, these geometric diodes rely on ballistic transport and are hence very reliant on high carrier mobilities.

Graphene is a 2D semi-metal where sp2 hybridisation causes an effectively linear energy-momentum dispersion relation, such that carriers behave as massless Dirac Fermions [15]. The lack of a bandgap has proven a barrier to 
(a)

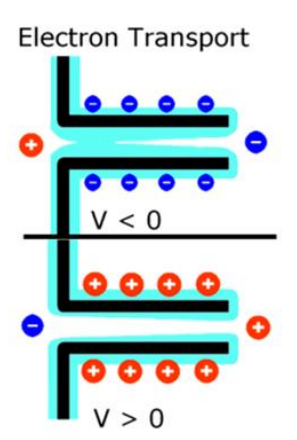

(b)

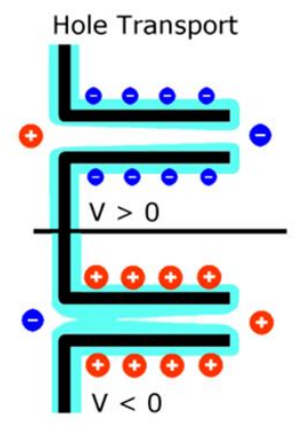

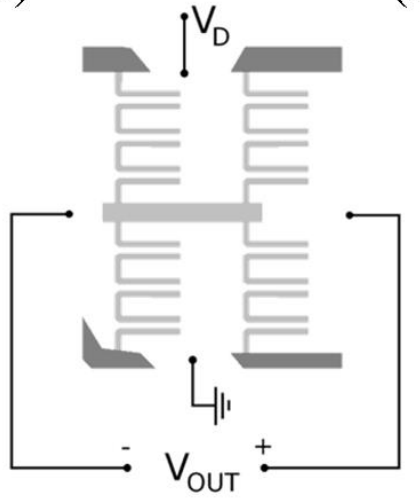

(c)

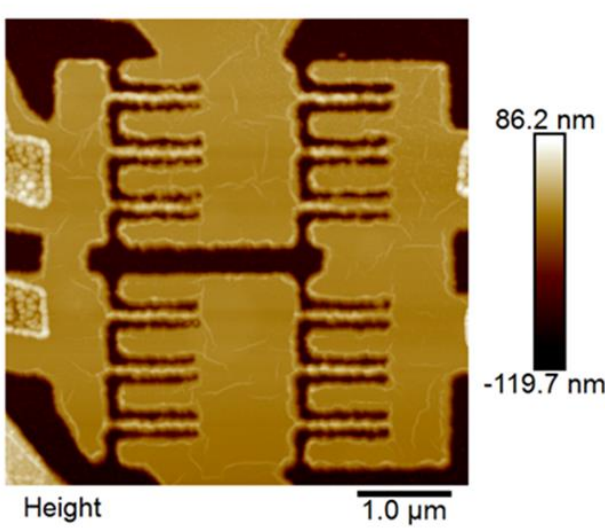

Figure 1. (a) The operational principle of an SSD, for the situations where electrons or when holes are the majority carrier. (b) Schematic of the fabricated GSSD bridge rectifier, with grey regions representing where the $\mathrm{hBN} / \mathrm{graphene} / \mathrm{hBN}$ is etched. (c) An AFM image of the finished device.

fully exploiting graphene's outstanding electrical properties, such as electrostatically tuneable carrier densities, high carrier velocities, and ultra-high carrier mobilities higher than $200,000 \mathrm{~cm}^{2} / \mathrm{Vs}$ [16-18]. However, the SSD does not necessarily require a bandgap to operate. Because of the lack of a bandgap and finite minimum conductivity, it is not possible to fully deplete and switch off the channel of a GSSD, causing larger reverse currents than SSDs formed from conventional semiconductor 2D electron gasses. Despite this, graphene's extremely high carrier mobilities and velocities, as well as its 2D nature, make it intrinsically suitable for fabrication of planar devices such as the SSD. The nature of 2D electronic devices such as the SSD makes them easy to upscale to large area production using patterning techniques such as imprinting, simplifying manufacture due to not needing to align multiple layers. The lack of a bandgap also results in the diode having zero threshold voltage [11].

The SSD operates by the electric field from 'side gates' either side of the channel altering the carrier density in the channel itself, causing the channel to be open under forward bias and closed under reverse bias. The channel and gates are separated by narrow trenches, as shown in figure 1(a), which can be patterned by techniques such as atomic force microscopy (AFM) lithography or electron-beam lithography (EBL). The 'forward' direction of the diode depends on the majority carrier, and in graphene the operational direction of the SSD switches when the device is biased to either side of the neutrality point (NP).

Despite the considerable amount of research performed on SSDs in various materials, there have been no published attempts to construct the simple full-wave rectifying structure known as the bridge rectifier. Here we present theory and measurements for such a device, fabricated using monolayer graphene encapsulated between thin hexagonal boron nitride ( $\mathrm{hBN}$ ) layers.

\section{Methods}

The $\mathrm{hBN}$ and graphene flakes were prepared by using the standard mechanical exfoliation method. An hBN/Gr/hBN stack was then formed, and transferred onto a $290 \mathrm{~nm}$ thick $\mathrm{SiO}_{2} / \mathrm{Si}$ substrate using a PMMA membrane. The patterns were formed using electron-beam lithography using a Zeiss EVO 10 with PMMA resist, with figure 1(b) showing the device design. The $\mathrm{hBN} / \mathrm{Gr} / \mathrm{hBN}$ stack was etched in a $\mathrm{CHF}_{3}$ and $\mathrm{O}_{2}$ mixure using an Oxford Instruments System 100 reactive ion etching system. For the contacts, onedimensional (1D) contacts were formed by first etching the exposed regions as described above, followed by depositing a $3 \mathrm{~nm}$ thick Cr layer and a $70 \mathrm{~nm}$ Au layer in sequence using electron-beam evaporation. By sweeping the back gate voltage applied to the $\mathrm{Si}$ susbtrate, the Dirac point (neutrality point) was found to be at $-8 \mathrm{~V}$, as shown in figure $2(\mathrm{a})$. The bulk field-effect mobility was found to be $28,232 \mathrm{~cm}^{2} / \mathrm{Vs}$ and $26,601 \mathrm{~cm}^{2} / \mathrm{Vs}$ for electrons and holes, respectively, from the slopes of the conductivity as a function of the back gate voltage. These mobilities were obtained from measurements of a Hall bar structure close to the GSSD bridge rectifier. Figure 2(b) shows the mobility as a function of gate voltage. An AFM image of the SSD bridge rectifier is shown in figure 1(c). The designed channel length and channel width are $1 \mu \mathrm{m}$ and $100 \mathrm{~nm}$, respectively. The width of the etched trenches is also designed to be $100 \mathrm{~nm}$ along the channel, to create the asymmetry, and $200 \mathrm{~nm}$ pependicular to the channel in order to reduce the parastic capacitance, hence improving the operating frequency. The devices were measured using Keithley 6487 source/measure unit, Keysight 34401A voltage meter, and Signal Recovery 7265 Lock-in amplifier in a cryostat system in vacuum.

\section{Theory and results}


2 (a)

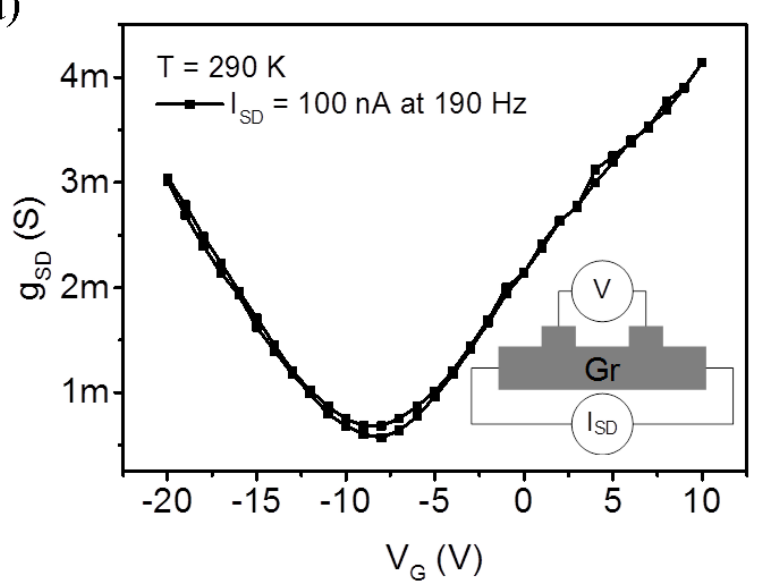

(c)

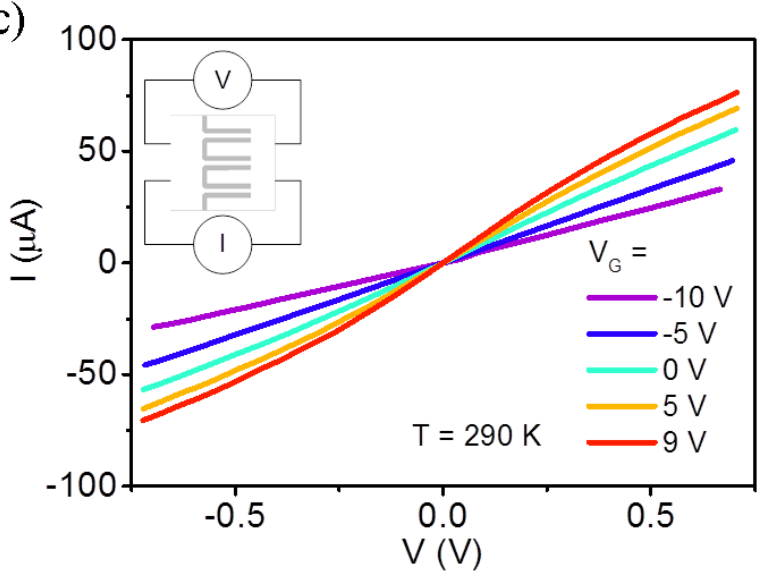

(b)

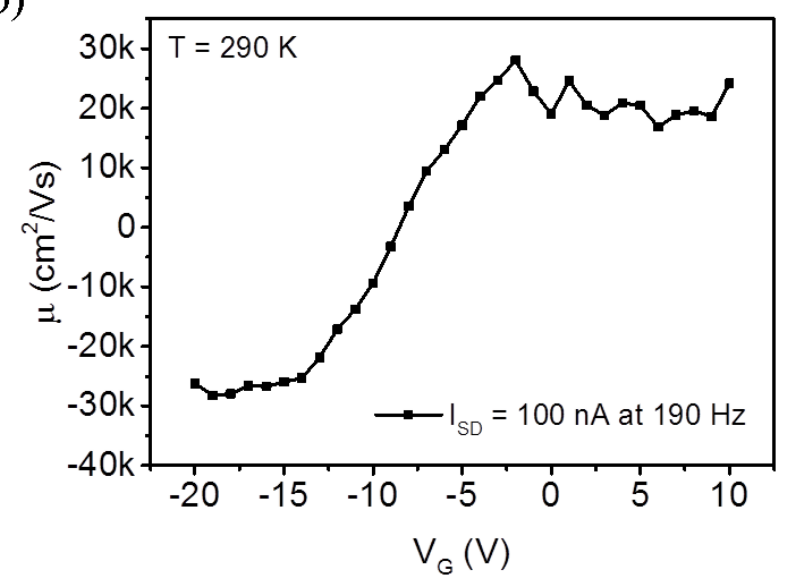

(d)

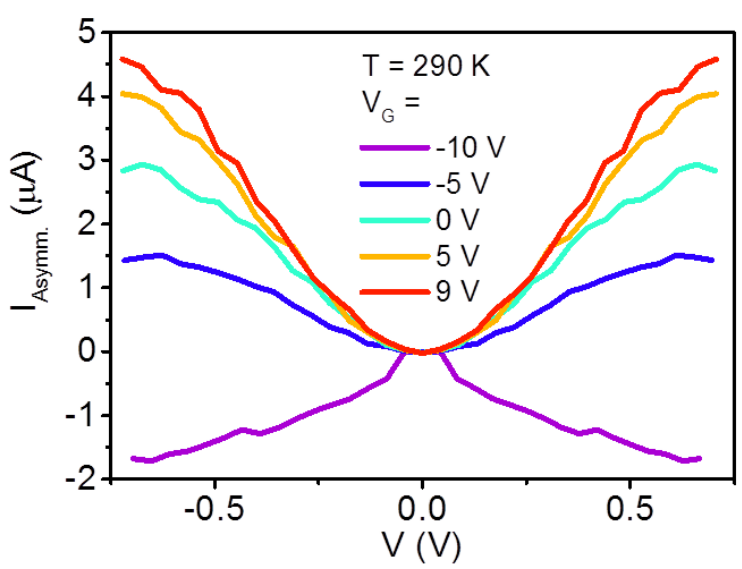

Figure 2: (a) Conductivity of the encapsulated graphene sample as a function of gate bias, showing the location of the neutrality point. (b) Carrier mobility as a function of gate bias. Negative values of mobility here represent hole conduction. (c) I-V characteristics of an array of three encapsulated graphene SSDs, at different gate biases. (d) Current asymmetry in the data shown in (c), showing the rectification capabilities of the graphene SSD array.

\subsection{Operation mechanism of a single SSD and of the bridge rectifier}

The current passing through a GSSD array can be derived by considering each SSD as a MOSFET with the drain and source connected using the Sichman-Hodges model $[11,19]$. The current $I_{G S S D}$ is given by

$$
I_{G S S D}=t_{c} W_{c} q n v,
$$

where $t_{c}$ and $W_{c}$ are the channel thickness and width respectively, $q$ is the electronic charge, and $n$ and $v$ are the carrier density and velocity respectively. The gate-induced carrier density is given by

$$
n=\frac{C_{A}}{q W_{c}}\left(V_{F}-V(y)\right),
$$

where $C_{A}$ is the trench capacitance per unit area, $V_{F}$ is the voltage difference between the drain voltage $V_{D}$ and the effective carrier neutrality point in the channel, and $V(y)$ represents the voltage as a function of distance along the channel. By expressing the drift velocity as $v=$ $-\mu d V(y) / d y$, where $\mu$ is the carrier mobility, and integrating along the channel, it is found that the current through an array of N SSDs is given by

$$
I_{G S S D}=\frac{N \mu C_{t}}{L}\left(V_{F} V_{D}+\frac{V_{D}^{2}}{2}\right),
$$

where $L$ is the channel length, and $C_{t}$ is the trench capacitance per unit length. In contrast to for a MOSFET, or indeed a semiconductor SSD, equation (3) contains no threshold voltage term. This is due to the semi-metal nature of graphene.

As the diodes on one side of the bridge rectifier have the same current passing through them, it can therefore be said that

$$
V_{F} V_{1}+\frac{V_{1}^{2}}{2}=V_{F} V_{2}-\frac{V_{2}^{2}}{2}
$$


(a)
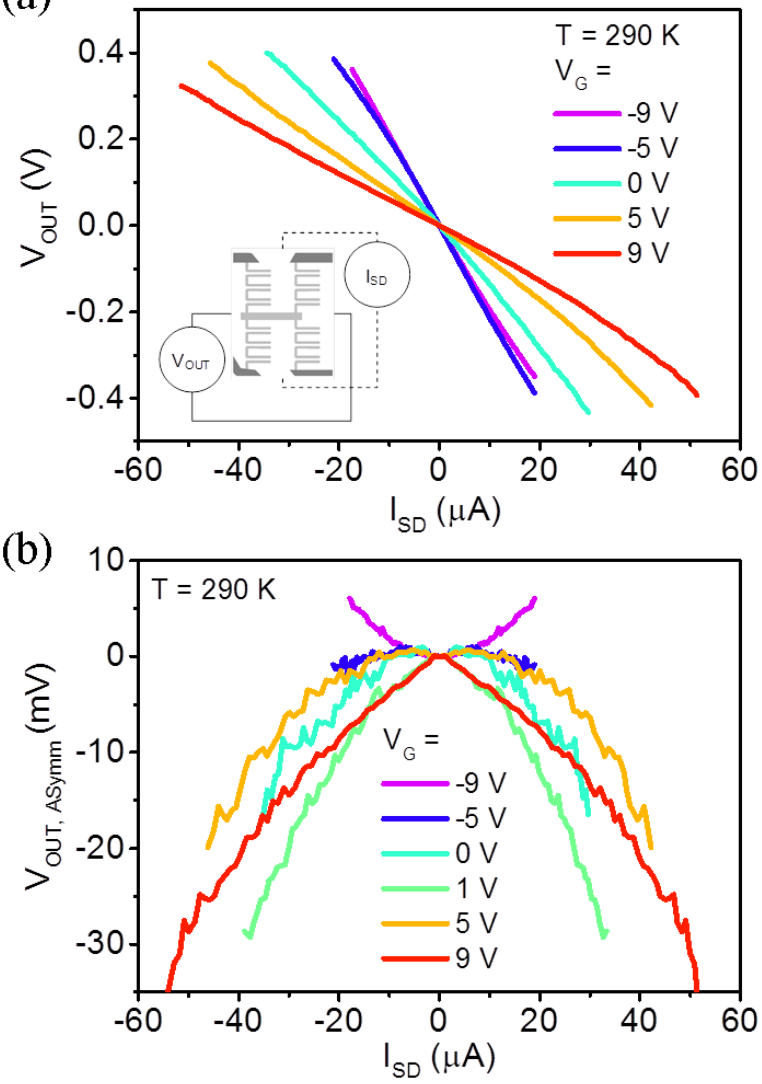

(c)

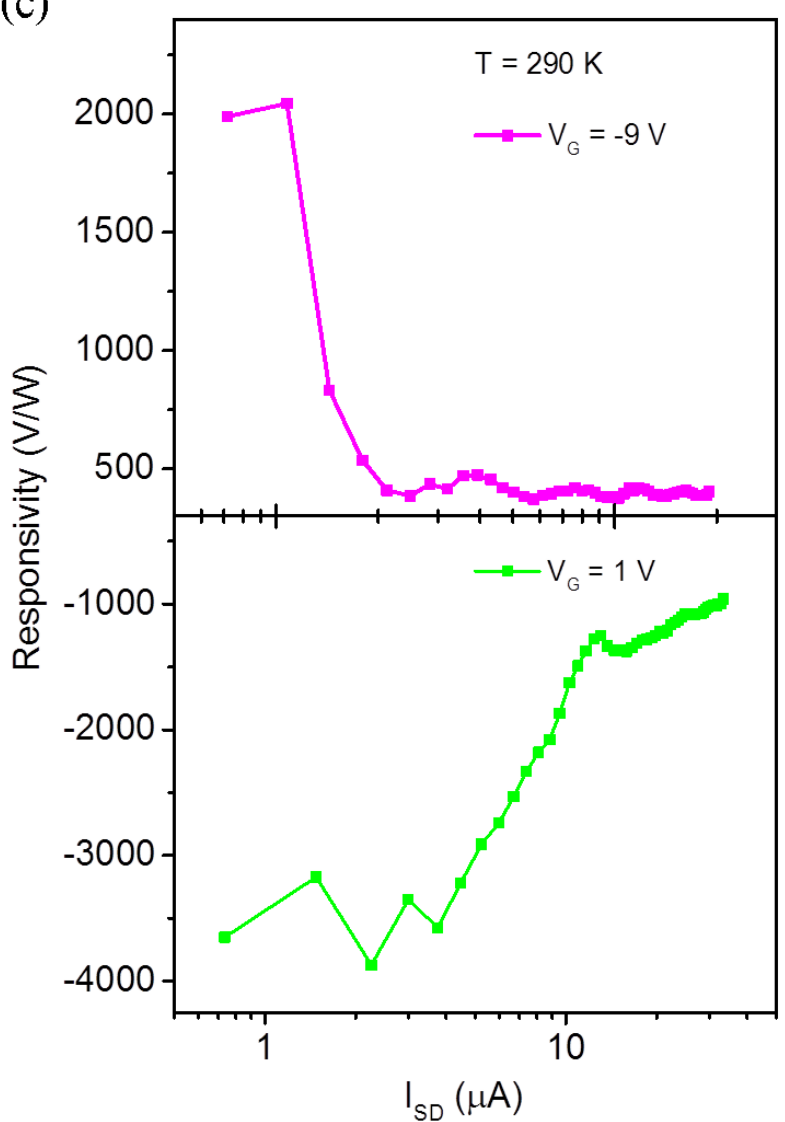

Figure 3: (a) Output voltage of the encapsulated graphene SSD bridge rectifier as a function of input current, at different gate voltages. The inset shows the device structure and measurement setup. (b) Asymmetry in the output voltage shown in (a). The switch in direction when the majority carrier switches from electrons to holes is visible, as is the low output in the vicinity of the neutrality point. (c) Responsivity of the bridge rectifier as a function of input current when holes are the majority carrier $\left(V_{G}=-9 \mathrm{~V}\right)$ and when electrons are the majority carrier $\left(V_{G}=1 \mathrm{~V}\right)$.

where $V_{1}$ and $V_{2}$ are voltage drops at the forward and the reverse biased diodes respectively. By assuming that each diode (SSD array) is identical, the voltage drops can be expressed in terms of their sum (the input voltage, $V_{i n}$ ) and their difference $\Delta V: V_{1}=\frac{V_{\text {in }}-\Delta V}{2}$ and $V_{2}=\frac{V_{\text {in }}+\Delta V}{2}$.

Substituting this into equation (4), it is found that

$$
\Delta V=2 V_{F} \pm \sqrt{4 V_{F}^{2}-V_{i n}^{2}} .
$$

It is worth noting that this equation is only valid for $V_{\text {in }} \ll$ $\left|V_{F}\right|$, and that the positive solution is non-physical. Taking the negative solution, a series expansion of this expression around $V_{\text {in }}=0$ gives

$$
\Delta V=\frac{V_{i n}^{2}}{4 V_{F}}+\frac{V_{i n}^{4}}{64 V_{F}^{3}}+O\left(V_{i n}^{6}\right) .
$$

The output voltage, $V_{\text {out }}$, is simply given by twice this voltage difference. The higher order terms drop off in magnitude extremely quickly, hence it is expected that

$$
V_{\text {out }} \approx \frac{V_{\text {in }}^{2}}{2 V_{F}} .
$$

Since the linear part of equation (3) dominates for GSSDs, and due to the symmetry of the bridge rectifier, the currentoperated output voltage is related to the input current by

$$
V_{\text {out }} \propto \frac{I_{S D}^{2}}{2 V_{F}^{3}} .
$$

This predicts a quadratic relationship between output voltage and input current, and an inverse cubic relationship between output voltage and back gate bias.

\subsection{Characteristics of Graphene SSDs}

The IV characteristics of three graphene SSDs are shown in figure 2(c). As expected there is no significant turn-on or threshold voltage observed, in contrast to conventional rectifiers using $\mathrm{p} / \mathrm{n}$ junctions or Schottky juntions. The nonlinear operation with zero threshold allows the device to have 
(a)

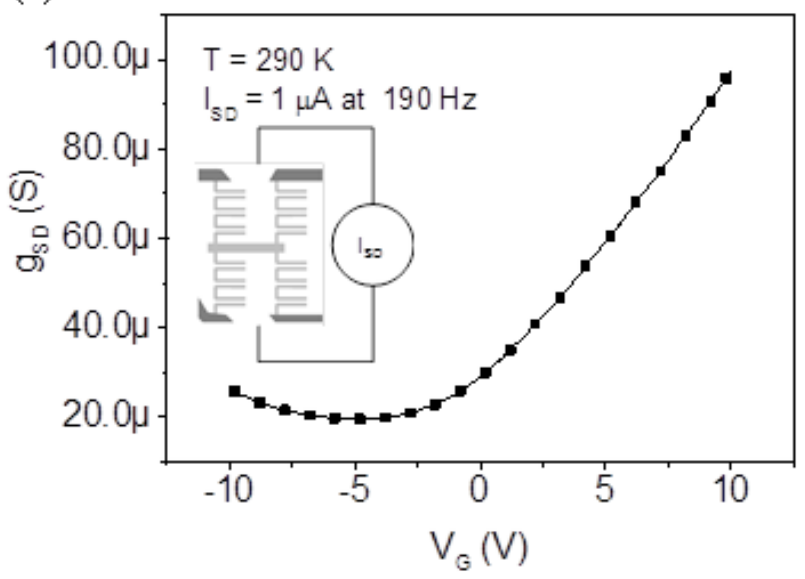

(c)

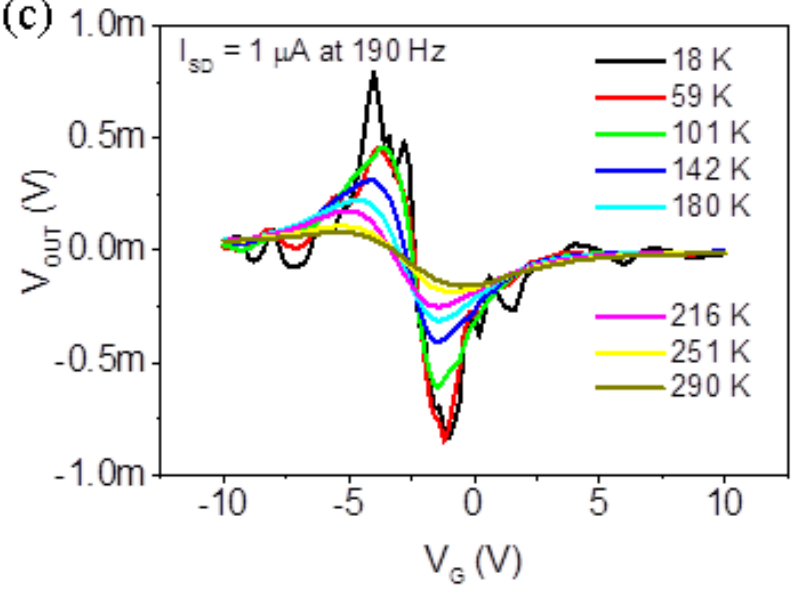

(b)

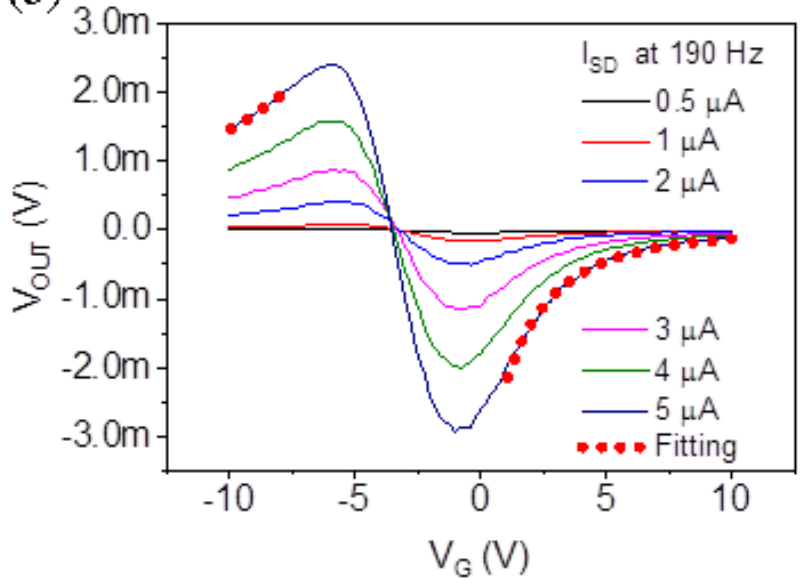

(d)

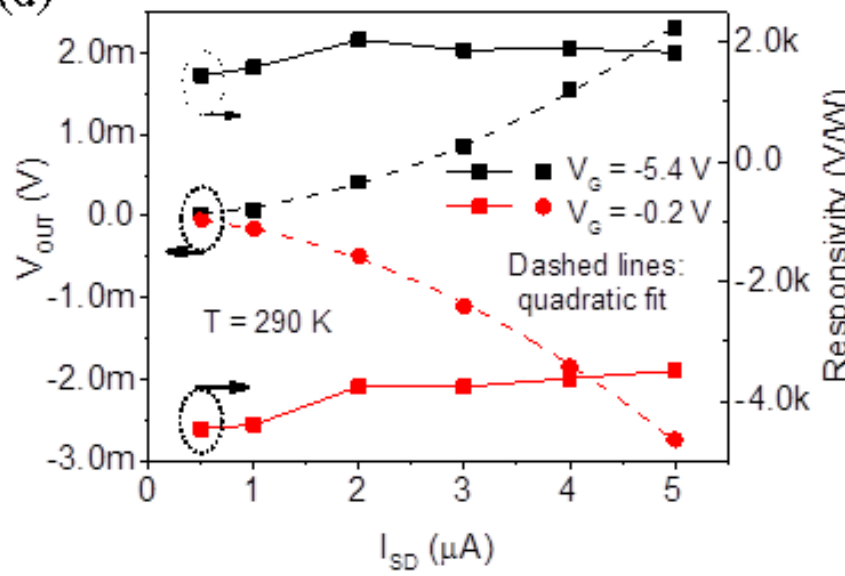

Figure 4: (a) Conductance of the graphene SSD bridge rectifier as a function of gate bias, with the inset showing the measurement setup. (b) DC output voltage as a function of gate bias, at different AC input currents at low frequency. The dashed line shows a $\propto V_{G}^{-3}$ fitting to the data for $5 \mu \mathrm{A}$, at gate biases far from the neutrality point. (c) DC output voltage as a function of gate bias at different temperatures, with an $\mathrm{AC}$ input current of $1 \mu \mathrm{A}$. (d) Room temperature DC output voltage (left) and responsivity (right) versus input current, at gate biases of $-5.4 \mathrm{~V}$ and $-0.2 \mathrm{~V}$. Dashed lines show

a decent current flow at a small bias. The resistance of the GSSD array at zero bias and zero gate voltage is $13.2 \mathrm{k} \Omega$. By using the theory proposed in ref. [11], the extrinsic in-plane capacitance of the SSD array is found to be $7.95 \times 10^{-18} \mathrm{~F}$. The intrinsic cut-off frequency, $f=1 /(2 \pi R C)$, is estimated to be $1.51 \mathrm{THz}$, making this device suitable to be used in $\mathrm{THz}$ detection.

For a sensing device, responsivity, one of the figures of merit, describes the voltage output gain of rectifiers. It can be estimated by using the zero-bias cuirvature coefficient, $\gamma=$ $\left(\frac{d^{2} I}{d^{2} V} / \frac{d I}{d V}\right)_{V=0}$. If the GSSD is connected to a RF source with an input impedance $\left(Z_{S}\right)$ of $50 \Omega$, the estimated responsivity, $\mathcal{R}$, is $43.3 \mathrm{~V} / \mathrm{W}$ by using $\gamma=0.433 \mathrm{~V}^{-1}$ and $\mathcal{R}=2 \operatorname{Re}\left(Z_{S}\right) \gamma$.

Figure 2(d) shows the asymmetric current of the SSD array, where

$$
I_{\text {Asymm. }}=\frac{I_{S D}\left(V_{D}\right)+I_{S D}\left(-V_{D}\right)}{2} .
$$

It demosntrates a nonlinearity at low biases which is quadratic, as described by equation (3). When the majority carriers change from holes to electrons, the polarity of the asymmetric current changes from negative to positive. This also agrees with the SSD theory, as is shown in figure 1(a). In the region around the NP, the presence of both charge carriers disrupts the SSD operation, causing the asymmetric current to fall to near zero, again as expected.

\subsection{IV characteristics of a graphene SSD bridge rectifier}

By cascading four GSSD arrays, a bridge rectifier can be built, which is shown in figure 1(c). The output voltage as a function of input DC current is shown in figure 3(a). However, it is difficult to observe the rectification behaviour 
as the linear component dominates the output voltage. In figure 3(b), the asymmetric output voltage,

$$
V_{\text {OUT,Asymm. }}=\frac{V_{O U T}\left(I_{S D}\right)+V_{O U T}\left(-I_{S D}\right)}{2}
$$

is plotted as a function of input current. This also changes quadratically with the input current, as is predicted by equation (7).

The voltage responsivity is defined as the output voltage per input power:

$$
\mathcal{R}=\frac{\text { output voltage }}{\text { input power }}
$$

Figure 3(c) shows the responsivity calculated from the output voltage and input power as a function of input DC current. At $V_{G}=-9 \mathrm{~V}$ and $-1 \mathrm{~V}$, the responsivity is found to be around $400 \mathrm{~V} / \mathrm{W}$ and $3,000 \mathrm{~V} / \mathrm{W}$ at $I_{S D}=5 \mu \mathrm{A}$, respectively.

In figure 4(a), the conductance between the source and drain as a function of the back gate is shown, with the inset showing the measurement setup. The minimum conductance is found to be at $V_{G}=-4.7 \mathrm{~V}$, indicating that this is the location of the Dirac point. This voltage is different to the NP of the bulk shown in figure 2(a), indicating that the channel has a different level of doping to the bulk.

The output voltage as a function of gate voltage is shown in figure 4(b). It is found that the output voltage changes from positive to negative at $V_{G}=-3.5 \mathrm{~V}$, which is close to the NP. When large $V_{G}$ takes the device far away from the $\mathrm{NP}$, it is found that $V_{\text {OUT }}$ is proportional to $\Delta V_{G}^{-3}$, shown as the dashed line in figure 4(b), where $\Delta V_{G}^{-3}$ is the gate voltage offset from the neutrality point. This confirms the predicted relationship shown in equation (8), with $V_{G}$ proportionally shifting the effective neutrality point in the GSSD channel and hence shifting $V_{F}$, the drain voltage-neutrality point offset. When $V_{G}$ is close to the NP point however, both electrons and holes exist in the channel. The output voltages from electrons and holes are in opposite directions, resulting in a decrease in $V_{\text {OUT }}$ as the NP point is approached. This distorts the dependance observed in equation (8).

Figure 4(c) shows the DC output voltage as a function of gate voltage at different temperatures. When decreasing the temperature, it is found that the output voltage increases. Such increase cannot be explained directly by equation (7), since the output voltage does not depend on the temperature nor the carrier mobility. By taking a close look at the curve, it is also noticed that the peak of output voltage moves closer to the NP point with decreasing temperautre. This indicates that the improvement of $V_{\text {OUT }}$ is caused by the carriers become less mixed at a lower temperature according to the Fermi distribution. This therefore reduces the effects of having carriers of opposite signs in the channel, hence increasing the output voltage. It is also found that the output voltage starts to fluctuate at $142 \mathrm{~K}$ and below. This may be caused by the surface traps and edge states that change the conductace in the narrow channels of the GSSDs.

In figure $4(\mathrm{~d})$, it is found that the output voltage increases quadratically with the input AC current while the responsivity remians the same. This quadratic relationship is as predicted by equation (8). At $V_{G}=-5.4 \mathrm{~V}$ and $-0.2 \mathrm{~V}$, the responsivity is $1,571 \mathrm{~V} / \mathrm{W}$ for holes and 4,395 V/W for electrons respectively, at $I_{S D}=1 \mu \mathrm{A}$. However, if this device were to be connected to an RF source with an input impedance, $Z_{S}$, of $50 \Omega$, the voltage responsivity is given by:

$$
\mathcal{R}_{50 \Omega}=\mathcal{R}\left(1-\Gamma^{2}\right),
$$

where $\Gamma=\frac{Z_{D}-Z_{S}}{z_{D}+Z_{S}}$ is the reflection coefficient between the SSD and the source and $Z_{D}$ is the resistance of the detector. When the resistance of the diode is much larger than $50 \Omega$ and at low frequencies,

$$
\mathcal{R}_{50 \Omega}=4 \mathcal{R} \frac{50 \Omega}{Z_{D}}
$$

For the graphene bridge rectifer, the resistance at $V_{G}=-5.4 \mathrm{~V}$ and $-0.2 \mathrm{~V}$ equal $50.8 \mathrm{k} \Omega$ and $35.5 \mathrm{k} \Omega$, corresponding to $\mathcal{R}_{50 \Omega}$ of $6.2 \mathrm{~V} / \mathrm{W}$ and $24.8 \mathrm{~V} / \mathrm{W}$, respectively.

Noise equivalent power (NEP), another figure of merit for detectors, describes the power of the lowest detectable signal. The NEP is defined as

$$
N E P=\frac{S_{V}}{\mathcal{R}}
$$

where $S_{V}$ is the voltage noise spectral density. At zero bias, the electrical noise of the bridge rectifier is dominated by the thermal noise. The voltage noise spectral density of the thermal noise equals $\sqrt{4 k_{B} T R}$, where $\mathrm{k}_{\mathrm{B}}$ is the Boltzmann constant, $\mathrm{T}$ is the temeprature and $\mathrm{R}$ is the resistance of the detector [6]. At $V_{G}=-5.4 \mathrm{~V}$ and $-0.2 \mathrm{~V}$, the NEP are $18.2 \mathrm{pW} / \mathrm{Hz}^{1 / 2}$ and $5.4 \mathrm{pW} / \mathrm{Hz}^{1 / 2}$, respectively. If the impedance mismatch with a $50 \Omega \mathrm{RF}$ source is also considered, the NEP increases to $4.61 \mathrm{nW} / \mathrm{Hz}^{1 / 2}$ and $0.96 \mathrm{nW} / \mathrm{Hz}^{1 / 2}$, respectively. Such performance is similar to that obtained by graphene ballistic rectifiers [20] and almost 10 times better than previous published results for graphene SSDs [9].

Considering the extrinsic in-plane capacitance of the GSSD bridge rectifier is only $7.97 \times 10^{-18} \mathrm{~F}$ and the resistance is $35.5 \mathrm{k} \Omega$, the cut-off frequency is estimated to be $0.56 \mathrm{THz}$ on a nonconductive substrate. The cut-off frequency can be further improved by increasing conductance between $\mathrm{S}$ and $\mathrm{D}$, and by minimizing extrinsic 
capacitance. The conductance can be increased by reducing the gaps between adjacent arrays in the bridge rectifier, hence reducing the spacing between $\mathrm{S}$ and $\mathrm{D}$. The extrinsic capacitance can be reduced by increasing the width of the trench perpendicular to the channel. This will cause a slight increase in S-D seperation and hence a slight decrease in conductance, however a three-fold increase in trench width will cut the extrinsic capacitance by a factor of three, while causing only a small decrease in conductance. Because of this, it is expected that these change will cause a considerable increase in cut-off frequency.

\section{Conclusion}

In summary, we have demonstrated a graphene SSD based bridge rectifier and studied its analytical theory. The measured IV characteristics are in good agreement with the proposed theory, with the output voltage varying as expected both as a function of input current and of gate bias. The peak intrinsic responsivities are 1,571 V/W for holes and $4,395 \mathrm{~V} / \mathrm{W}$ for electrons with an input current of $1 \mu \mathrm{A}$ at 190 $\mathrm{Hz}$. Under these conditions, the noise equivalent powers for holes and electrons are calculated to be $18.2 \mathrm{pW} / \mathrm{Hz}^{1 / 2}$ and $5.4 \mathrm{pW} / \mathrm{Hz}^{1 / 2}$ respectively. By using the calculated extrinsic capacitance and the device resistance, the instrinsic cut-off frequencies of a SSD array and bridge rectifier are estimated to be $1.51 \mathrm{THz}$ and $0.56 \mathrm{THz}$, respectively, making the graphene SSDs and bridge recitfiers suitable to be used for $\mathrm{THz}$ detection. By making modifications to device designs, it is expected that these frequencies can be substantially improved, pushing the operation of this device further into the $\mathrm{THz}$ region.

\section{Acknowledgements}

We thank M. McGowen, I. Hawkins, and L. Zhang for technical support. This work was supported by the NorthWest Nanoscience Doctoral Training Center, EPSRC Grants EP/N021258/1, EP/G03737X/1, and EP/L01548X/1, National Key Research and Development Program of China Grants 2016YFA0301200 and 2016YFA0201800, and National Natural Science Foundation of China Grants 11374185 and 11304180 .

\section{References}

[1] Song A M, Missous M, Omling P, Peaker A R, Samuelson $\mathrm{L}$ and Seifert W 2003 Unidirectional electron flow in a nanometer-scale semiconductor channel: A self-switching device Appl. Phys. Lett. 83 1881-3

[2] Song A M, Maximov I, Missous M and Seifert W 2004 Diode-like characteristics of nanometer-scale semiconductor channels with a broken symmetry Phys. E Low-Dimensional Syst. Nanostructures 21 1116-20

[3] Pardo D, Mateos J, Song A M, Vasallo B G and Gonzalez T $2005 \mathrm{THz}$ operation of self-switching nano-diodes and nano-transistors Nanotechnol. II 5838145
[4] Kasjoo S R and Song A M 2013 Terahertz detection using nanorectifiers IEEE Electron Device Lett. 34 1554-6

[5] Daher C, Torres J, Iñiguez-de-la-Torre I, Nouvel P, Varani L, Sangaré P, Ducournau G, Gaquière C, Mateos J and González T 2016 Room temperature direct and heterodyne detection of 0.28-0.69-THz Waves Based on GaN 2-DEG unipolar nanochannels IEEE Trans. Electron Devices 63 $353-9$

[6] Winters M, Thorsell M, Strupiński W and Rorsman N 2015 High frequency electromagnetic detection by nonlinear conduction modulation in graphene nanowire diodes Appl. Phys. Lett. 107

[7] Majewski L A and Song A M 201620 Megahertz Operation of Organic Nanodiodes Phys. Status Solidi Basic Res. 253 1507-10

[8] Inoue M, Kasai S, Maemoto T, Kimura Y, Sun Y and Sasa S 2013 Rectification Effects of ZnO-Based Transparent Nanodiodes on Glass and Flexible Plastic Substrates Jpn. J. Appl. Phys. 52 06GE09

[9] Westlund A, Winters M, Ivanov I G, Hassan J, Nilsson PÅ, Janzén E, Rorsman N and Grahn J 2015 Graphene selfswitching diodes as zero-bias microwave detectors Appl. Phys. Lett. 106093116

[10] Al-Dirini F, Skafidas E and Nirmalathas A 2013 Graphene Self Switching Diodes with high rectification ratios Proc. IEEE Conf. Nanotechnol. 698-701

[11] Singh A K, Auton G, Hill E and Song A 2018 Estimation of intrinsic and extrinsic capacitances of graphene selfswitching diode using conformal mapping technique $2 D$ Mater. 5035023

[12] Al-Dirini F, Hossain F M, Nirmalathas A and Skafidas E 2014 All-graphene planar self-switching MISFEDs, metalinsulator-semiconductor field-effect diodes Sci. Rep. 4 3983

[13] Ghaziasadi H, Jamasb S, Nayebi P and Fouladian M 2018 Rectification of graphene self-switching diodes: Firstprinciples study Phys. E Low-Dimensional Syst. Nanostructures 99 123-33

[14] Moddel G, Zhu Z, Grover S and Joshi S 2012 Ultrahigh speed graphene diode with reversible polarity Solid State Commun. 152 1842-5

[15] Novoselov K S, Geim A K, Morozov S V., Jiang D, Katsnelson M I, Grigorieva I V., Dubonos S V. and Firsov A A 2005 Two-dimensional gas of massless Dirac fermions in graphene Nature $\mathbf{4 3 8}$ 197-200

[16] Novoselov K S, Geim A K, Morozov S V., Jiang D, Zhang Y, Dubonos S V., Grigorieva I V. and Firsov A A 2004 Electric Field Effect in Atomically Thin Carbon Films Science 306 666-9

[17] Bolotin K I, Sikes K J, Jiang Z, Klima M, Fudenberg G, Hone J, Kim P and Stormer H L 2008 Ultrahigh electron mobility in suspended graphene Solid State Commun. 146 $351-5$

[18] Auton G, Zhang J, Kumar R K, Wang H, Zhang X, Wang Q, Hill E and Song A 2016 Graphene ballistic nanorectifier with very high responsivity Nat. Commun. 7 11670

[19] Shichman H and Hodges D A 1968 Modeling and Simulation of Insulated-Gate Field-Effect Transistor Switching Circuits IEEE J. Solid-State Circuits $3285-9$

[20] Auton G, But D B, Zhang J, Hill E, Coquillat D, Consejo C, Nouvel P, Knap W, Varani L, Teppe F, Torres J and Song A 2017 Terahertz Detection and Imaging Using Graphene Ballistic Rectifiers Nano Lett. 17 7015-20 